\title{
ZUR ONTOLOGIE NATÜRLICHER ARTEN
}

\author{
Christian Kanzian
}

UDK 111

\section{Die nominalistische und die realistische Verführung}

Das Thema "Ontologie natürlicher Arten" ist verführerisch. Nicht wenige Philosophen verführt es zu spontanen Abwehrreaktionen: Die Akzeptanz natürlicher Arten ist schlechte Metaphysik, die als Resultat einer schlechten Semantik bestimmter (sortaler) Termini zu erklären ist. Es gibt nur einzelne, konkret vorkommende Individuen. Somit hat es überhaupt keinen Sinn, in eine Ontologie irgendwelche Arten, schon gar nicht die sogenannten "natürlichen", einzuführen. Der Nachteil dieser "nominalistischen Verführung" besteht darin, daß sie, ohne zu zögern, in zentralen Fragen der Ontologie, wie es z. B. das Universalienproblem eine ist, zu Extrempositionen verleitet. Diese stehen ihrerseits bekanntermaßen auf schwachen Füßen. Armstrongs Problem-Exposition sei nur als eine Möglichkeit angeführt, ihre Schwächen deutlich zu machen. ${ }^{1}$

Bei anderen Autoren ruft das Thema einige Euphorie hervor: Wir können nicht alle Aussagen über Arten vollständig in Aussagen über konkrete Vorkommnisse dieser Arten übersetzen. Deshalb haben wir anzunehmen, daß es nicht nur konkrete Vorkommnisse gibt, sondern auch die Arten, denen sie angehören. Es gibt nicht nur konkrete Katzen und Hunde, sondern auch die Spezies Katze, die Art Hund. Manche Realisten erachten Arten sogar als Paradebeispiele zeitloser oder ewiger Entitäten, die unabhängig von konkreten Vorkommnissen existieren. Philosophen, die dieser "realistischen Verführung” zum Opfer fallen, sehen sich Vorwürfen ausgesetzt, denen alle (extrem) universalien-realistische Positionen ausgesetzt sind. Sie haben, wie Quine zu sagen pflegt, eben keinen Sinn für die "Schönheit ontologischer Wüstenlandschaften". ${ }^{2}$

Ich möchte hier nicht die Kritik an Opfern der genannten Verführungen explizieren. Ich stelle mir vielmehr die Frage, ob man das Problem einer On- 
tologie natürlicher Arten auf eine Weise angehen kann, die nicht von vornherein dem Verdacht ausgesetzt ist, einer der genannten Verführungen erlegen zu sein. In meinem Beitrag möchte ich Voraussetzungen einer solchen Vorgangsweise andeuten (Punkt 1 und 2). In der Folge (Punkt 3 und 4) versuche ich eine Annäherung an den Begriff "natürliche Arten". Diese Annäherung kommt zu einer inhaltlichen Option: Die Rede von natürlichen Arten ist ontologisch unverzichtbar, ohne auf extrem realistische Deutungen natürlicher Arten festzulegen. Mit der Anführung dieser Option verbinde ich keineswegs den Anspruch, das Problem einer Ontologie natürlicher Arten zu lösen; eher den, zur weiteren Aktualisierung seiner Diskussion beizutragen.

\section{Die ontologische Konstitution von Dingen}

Die z. Z. gängigste Formulierung eines der grundlegendsten Axiome der Ontologie wird auf ein Diktum Quines zurückgeführt. Es besagt, daß es keine Entität geben kann ohne Identität. Auf gut Deutsch: No Entity without Identity. Die Frage nach dem, was bestimmte Entitäten zu dem macht, was sie ontologisch betrachtet sind, kann demnach auch als die Frage gestellt werden, was bestimmte numerische Einheiten als solche konstituiert. Was aber ist das, was Dinge als solche ontologisch konstituiert? Was macht ein Ding zu einer numerischen Einheit?

Ein erster Ansatz zu einer Antwort kann in der Feststellung bestehen, daß Dinge raum-zeitliche Vorkommnisse sind. Das bedeutet, daß man ohne die Angabe einer raum-zeitlichen Position nicht angeben kann, was ein Ding ontologisch konstituiert. Weiterhin ist es selbstverständlich, daß ein und dasselbe Ding ein und dieselbe Raum-Zeit-Position einnimmt. Stimmen Gegenstände in raum-zeitlichen Eigenschaften nicht überein, wird man von ihnen nicht als von numerischen Einheiten sprechen können. Im Anschluß daran stellt sich die Frage, ob die ontologische Konstitution von Dingen, ihre numerische Identität, über einen Verweis auf raum-zeitliche Positionen vollständig zu klären ist. Ist die raum-zeitliche Übereinstimmung ("Koinzidenz”) nicht nur notwendige, sondern auch hinreichende Identitätsbedingung von Dingen? Gehen Dinge ontologisch betrachtet darin auf, Raum-Zeit-Zonen zu sein? Will man diese Fragen positiv beantworten, hat man u. a. zu zeigen, daß Dinge, die sich vollständig raum-zeitlich überlappen, in jedem Fall identisch sind. Man hat auszuschließen, daß numerisch verschiedene Dinge zur gleichen Zeit am selben Ort vorkommen können.

Vor dem Hintergrund alltäglicher Überzeugungen bezüglich Dingen stellen sich einige Zweifel an der allgemeinen Gültigkeit dieser These ein. Nach der Möglichkeit der völligen Überlappung von Dingen gefragt, fallen uns spontan eine Reihe von Beispielen ein. Interessant an ihnen ist, daß sie vorerst 
zu keinem eindeutigen Ergebnis führen. Natürlich kommt es uns seltsam vor, davon zu sprechen, daß zwei Bäume zur selben Zeit am gleichen Ort sind. Aber: Hat es nicht doch Sinn anzunehmen, daß ein Stück Holz und ein Baum exakt dieselbe Raum-Zeit-Karriere haben können, ohne sich darauf festzulegen, daß es sich bei Holz und Baum um ein und dasselbe handelt? Es erscheint uns als unsinnig anzunehmen, daß sich zwei Menschen zur selben Zeit am gleichen Ort befinden. Aber: Stellt sich nicht ein gewisses Unbehagen ein, wenn wir von vornherein in Abrede stellen, daß ein Mensch und die Summe seiner körperlichen Bestandteile nicht ein und dasselbe sind, obwohl sie exakt zur gleichen Zeit am selben Ort vorkommen? Eine erste Regelmäßigkeit ergibt sich aus folgenden Feststellungen: Beispiele numerisch verschiedener Vorkommnisse, von denen wir intuitiv geneigt sind zu bezweifeln, daß sie sich raum-zeitlich vollständig überlagern können, gehören derselben Art an. Numerisch verschiedene Vorkommnisse, die zur selben Zeit am gleichen Ort sind, gehören hingegen unterschiedlichen Arten an. Offensichtlich ist, zumindest alltäglichen Intuitionen nach, für die Erörterung der Identität von Dingen neben ihrer raum-zeitlichen Position auch ihre Artzugehörigkeit zu berücksichtigen.

Derartige Intuitionen finden im fachphilosophischen Diskurs ihren Niederschlag. Weit verbreitet ist die Skepsis gegenüber der Auffassung, die raumzeitliche Koinzidenz sei nicht nur notwendige, sondern auch hinreichende Identitätsbedingung von Dingen. ${ }^{3}$ Es war nicht zuletzt die Skepsis gegenüber der Auffassung, daß Dinge als Raum-Zeit-Zonen in hinreichender Weise ontologisch zu bestimmen sind, welche der These von der sortalen Abhängigkeit oder Dependenz der Identität von Dingen neu zur Geltung verholfen hat. ${ }^{4}$ Es würde von unserem Thema zu weit weg führen, diese Diskussion auch nur ansatzweise darzustellen oder gar selbst eine Begründung zu versuchen. Wir müssen uns damit begnügen, die sortale Abhängigkeit der Identität von Dingen als eine (vor dem Hintergrund alltäglicher Intuitionen plausible und durch eine Reihe namhafter Autoren gestützte) Voraussetzung für das folgende anzunehmen.

\section{Die sortale Dependenz der Identität von Dingen}

Um die Relevanz der These von der sortalen Dependenz der Identität von Dingen für unser Thema zu verdeutlichen, hat man zu erläutern, worin die

Belege und Argumente dafür führt u. a. an: Kanzian 1994.

4 Vgl. dazu v. a. Wiggins 1980, aber auch Hirsch 1982, v. a. chapt. 2, sowie Lowe 1989, u. a. 5 . 
besagte Abhängigkeit genauerhin besteht. ${ }^{5}$ Eine Weise, diese Abhängigkeit zu erörtern, ist, ontologische Bedingungen zu analysieren, denen Dinge unterliegen, insofern sie den jeweiligen Arten angehören.

Eine erste Gruppe ${ }^{6}$ solcher Bedingungen möchte ich "Existenzbedingungen” nennen. Daß mit der sortalen Zugehörigkeit von Dingen Bedingungen ihrer Existenz gekoppelt sind, besagt zunächst, daß die Existenz jedes Dinges davon abhängt, daß es auf irgendeine Weise irgendeiner Art angehört. Neben dieser sehr allgemeinen Feststellung ist noch zu berücksichtigen, daß mit Arten besondere Bedingungen der Existenz für die ihnen zugehörigen Vorkommnisse gekoppelt sind. Eine zweite Gruppe sind Kontinuitätsbedingungen. Diese betreffen nicht nur die (räumliche) Ausdehnung von Individuen zu einem bestimmten Zeitpunkt. Sie sind auch für ihre Existenz durch die Zeit maßgeblich. Mit Kontinuitätsbedingungen sind Bedingungen des zeitlichen Beginns eines Individuums ebenso gekoppelt wie solche seines zeitlichen Endes sowie Bedingungen seines zeitlichen Verlaufs. Als dritte Gruppe seien Konstitutionsbedingungen angeführt. Sie geben Auskunft über den mereologischen Bestand: Ob Dinge, die dieser oder jener Art angehören, aus gleichartigen oder verschiedenen Teilen bestehen. Weiterhin darüber, welche Teile eines Individuums für seine Artzugehörigkeit notwendig sind. Zu Konstitutionsbedingungen gehören auch Bedingungen der materiellen Konstitution von Dingen.

Im folgenden frage ich mich, ob es nicht Entitäten gibt, deren Vorkommen auf besondere Weise sortal abhängig ist. Das tue ich, der Anschaulichkeit halber, anhand eines konkreten Beispiels: Ich erwäge, ob bzw. inwiefern es Unterschiede für ein Vorkommnis ausmacht, der Art Kuh bzw. der Art Computer anzugehören. Dabei stelle ich folgende These zur Diskussion: Der Unterschied zwischen der sortalen Dependenz der Identität einer Kuh und jener der Identität eines Computers ist anhand der Differenz, wie diese artspezifischen Existenz-(3.1), Kontinuitäts-(3.2) sowie Konstitutionsbedingungen (3.3) unterliegen, darzulegen. Zuvor möchte ich jedoch an die Bemerkung in der Einleitung anschließen und bekunden, daß es mir hier keineswegs um dogmatische Festlegungen, sondern um die Einladung zu einer Diskussion geht.

Weiterhin hätte man der Frage nachzugehen, welche Arten es sind, die für derartige Überlegungen überhaupt in Frage kommen. Auch dies würde den Rahmen dieses Beitrags überschreiten. Nur eine kurze Andeutung: Es sind jene Arten, für die keine spezielleren Unterarten angegeben werden können ("species infimae"). Zu deren genaueren Bestimmung verweise ich auf Hirsch 1971, u. a. 35, oder Lowe 1989, 40.

6 Diese Unterteilung in die drei genannten Gruppen erhebt weder den Anspruch, ausschließend noch den, exhaustiv zu sein. 


\section{Rosa und Robert}

3.1 Beginnen wir mit Robert, meinem Personalcomputer. Es ist nicht schwer einzusehen, daß Robert existiert, solange er als Computer existiert. Solange er als solcher funktioniert, haben wir keine Veranlassung, davon zu sprechen, daß es ihn nicht mehr gibt. Nehmen wir nun aber an, ich möchte Robert nicht weiter als Computer verwenden und demontiere seine wesentlichen Funktionseinheiten. Offensichtlich beende ich damit Roberts Existenz als Computer. Nehmen wir weiterhin an, ich führe Robert nun einer anderen Verwendung zu und mache aus ihm einen Stellplatz für eine gute alte Schreibmaschine, eine Unterlage für einen Blumenständer oder einfach ein Kunstobjekt. Wir können nun nicht mehr davon sprechen, daß Robert weiter ein Computer ist. Was hindert uns jedoch daran anzunehmen, daß Robert als Individuum (einer anderen Art) weiter existiert? Berücksichtigt man derartige Überlegungen, erscheint es als fraglich, ob es sich bei den mit der Art Computer gekoppelten Existenzbedingungen um notwendige Bedingungen für die Existenz eines Individuums als Individuum handelt. Und zwar deshalb, weil nichts dagegenspricht, durch funktionale oder andere pragmatische Festlegungen die Existenz des Dinges gleichsam über sein Computer-sein hinaus zu verlängern.

Fassen wir nun eine Kuh, Rosa, ins Auge. Gilt das, was eben von Robert gesagt wurde, in derselben Weise auch von Rosa? Auch bei Rosa ist es nicht schwer einzusehen, daß sie existiert, solange sie als Kuh existiert. Solange sie ihr Kuhleben führt, haben wir keine Veranlassung, davon zu sprechen, daß es sie nicht mehr gibt. Nehmen wir aber an, Rosas Kuhleben nimmt sein seliges Ende. Hat es Sinn, davon zu sprechen, daß das, was wir von Rosa nun einer anderen Verwendung zuführen, als dasselbe Individuum zu betrachten ist wie das, welches tags zuvor noch auf der Weide herumspaziert ist? Hat es Sinn davon zu sprechen, daß es dasselbe ist, nur eben jetzt ein Vorkommnis einer anderen Art? Sprechen nicht gute Intuitionen dafür, daß Rosa als Kuh existiert oder eben gar nicht existiert; dafür, daß die Zugehörigkeit zur Art Kuh eine notwendige Bedingung für ihre Existenz als Individuum ist? Sprechen nicht gute Intuitionen dagegen, daß wir die Existenz Rosas nach funktionalen oder anderen pragmatischen Kriterien einfach über ihr Kuh-sein hinaus verlängern könnten?

Natürlich könnte man einwenden, daß die hier angedeutete Unterscheidung zwischen Kühen und Computern "nur" auf Intuitionen beruht. — Selbst wenn man dem Einwand konzediert, daß durch die angedeutete Unterscheidung die für uns relevante Eigenart von Individuen der Art Kuh gegenüber jenen der Art Computer nicht in einem engen Sinn zu beweisen ist, kann der Verweis auf die genannten Intuitionen dazu beitragen, demjenigen, der die angesprochene Differenz leugnet, eine gewisse Beweislast aufzubürden. Er 
hätte entweder zu erweisen, daß auch die Existenz von Individuen wie Robert notwendig von ihrer Artzugehörigkeit abhängt; oder aber darzulegen, unter welcher Rücksicht man Individuen wie Rosa als numerisch identische Vorkommnisse wechselnder Arten auffassen kann.

3.2 Bei der Diskussion von Kontinuitätsbedingungen möchte ich mich zunächst auf einen Aspekt von Roberts zeitlicher Karriere konzentrieren. Und zwar den, daß nichts dagegenspricht, sie einfach zu unterbrechen. Nichts hindert einen Fachmann, Robert vollständig zu zerlegen und ihn nach einer beliebigen Zeit wieder zusammenzusetzen. Nichts spricht gegen die Behauptung, Robert existiere in der Zeit zwischen Zerlegung und Zusammenfügung nicht. Ebensowenig spricht dagegen, ihn nach erfolgreicher Zusammenfügung als denselben Computer zu erachten wie vor der Zerlegung. Vor dem Hintergrund der hier eingeführten Terminologie ist diese Feststellung dahingehend zu deuten, daß mit der Art Computer Kontinuitätsbedingungen gekoppelt sind, die zeitliche Unterbrechungen der durch sie betroffenen Individuen zulassen. Außerdem lassen sich, unter Berücksichtigung u. a. funktionaler Gesichtspunkte, Kontinuitätsbedingungen der Art Computer beliebig abändern. So sind z. B. Bedingungen, welche die räumliche Ausdehnung betreffen, unter dieser Rücksicht zu variieren. Früher war die räumliche Ausdehnung eines PCs durch die seiner maßgeblichen Funktionseinheiten sowie Bildschirm, Tastatur etc. bestimmt. Heute kann man durchaus annehmen, daß auch Teile von Großrechnern zur Extension von Personalcomputern gezählt werden können; z. B. jene Teile, an die PCs zwecks Zugangs zu Programmen, Datenträgern oder zur Teilnahme am Internet angeschlossen sind.

Bei Rosa ist festzuhalten, daß ihr Vorkommen nicht oder zumindest nicht so wie das Roberts unterbrochen werden kann. Zerlege ich Rosa wie Robert, ist es endgültig um sie geschehen. Die mit der Art Kuh gekoppelten Kontinuitätsbedingungen lassen keine Unterbrechungen der Existenz der ihr zugehörigen Individuen zu. Genausowenig erscheint es als plausibel, daß jene Bedingungen, denen der zeitliche Verlauf oder die räumliche Ausdehnung von Kühen unterliegen, nach funktionalen Gesichtspunkten variiert werden könnten.

Gegen den ersten Punkt kann angeführt werden, daß es zwar faktisch nicht möglich ist, Kühe unter dieser Rücksicht Computern gleich zu behandeln. Als Gedankenexperiment sei dies jedoch durchaus möglich. Gute science-fiction Filme operieren ja damit. Denken wir ans "Beamen", die zeitlich befristete Atomisierung, die sowohl von Computern als auch von Kühen möglich ist. Dieser Vorgang gehört im guten alten Raumschiff Enterprise zur alltäglichen Routine. - Aus derartigen Gedankenexperimenten, so könnten wir uns verteidigen, ist allerdings kein Argument gegen unsere Differenzierung zu gewinnen. Und zwar deshalb nicht, weil ihre Denkbarkeit bereits eine Ent- 
scheidung, und zwar eine negative, in dieser Frage voraussetzt: Der Gedanke, daß man, ich bleibe beim Beispiel, Kühe beamen kann, setzt voraus, daß man sie unter der fraglichen Rücksicht wie (komplexere) Computer betrachtet.

Gegen den zweiten Aspekt ist vorzubringen, daß wir letztlich doch auch für Kühe beliebige alternative Kontinuitätsbedingungen konstruieren könnten. Was hindert uns, aus pragmatischen Gesichtspunkten, zur Ausdehnung von Kühen z. B. auch deren Platz im Kuhstall zu rechnen? - Auch hier müssen wir nicht darauf beharren, aus den angeführten Beispielen einen "Beweis" für den Unterschied zwischen Kühen und Computern erbracht zu haben. Es mag der Hinweis genügen, daß auch der Leugnung der hier ins Auge gefaßten Differenz einiger Erklärungsbedarf erwächst; nicht zuletzt angesichts höchst gegenintuitiver Befunde beim Versuch, Kühe auch hier mit Computern gleichzuschalten.

3.3 Vermutlich gibt es in der Computertechnik einen Konsens in der Frage, welche Teile ein Individuum aufweisen muß, will es der Art Computer angehören. Wahrscheinlich aber auch darin, daß sich Art und Anzahl dieser Teile in der (kurzen) Geschichte der Computertechnologie geändert haben und sicherlich noch ändern werden. Dasselbe gilt, vermutlich noch augenfälliger, für die materielle Konstitution von Computern. Aus welchen Materialien PCs in 10, 20 oder 50 Jahren bestehen werden, wird sich heute kaum jemand zu prognostizieren wagen.

Auch hierin unterscheiden sich Kühe von Computern. Fachleute werden jene Teile genau angeben können, welche für die Zugehörigkeit von Individuen zur Art der Kühe notwendig sind. Wahrscheinlich gibt es aber auch darin einen Konsens, daß sich Art und Anzahl dieser Teile seit dem Bestehen der Spezies nicht geändert haben und auch nicht ändern werden. Dasselbe gilt, noch deutlicher, für die materielle Konstitution von Kühen.

Gegen diese Unterscheidung könnte ins Treffen geführt werden, daß sich auch Arten wie die der Kühe ändern. Sei es langsam, durch Evolution; sei es schneller, z. B. durch gentechnische Manipulation. Somit können wir hinsichtlich der mit der Art der Kühe gekoppelten Konstitutionsbedingungen keine prinzipiellen Unterschiede zu Compu tern feststellen. - Dem ist entgegenzuhalten, daß man den Fall einer derart gravierenden evolutionären Entwicklung, wie sie bei der Änderung der angesprochenen Konstitutionsbedingungen vorliegt, wohl eher als Entstehen einer neuen Spezies zu deuten hat denn als Änderung einer bestehenden. Die durch den Einwand selbst vorgenommene (problematische) Anlehnung des ontologischen Begriffs "Spezies" an den der Biologen erhöht sogar die Plausibilität dieser Einschätzung. Auch aus der Feststellung, daß man Vorkommnisse der Art Kuh künstlich verändern könnte, läßt sich kein Argument gegen unsere Differenzierung ableiten. Manipulieren wir Kühe so geringfügig, wie das z. Z. wohl 
noch geschieht, betrifft das nicht ihre artspezifischen Konstitutionsbedingungen. Sollten Biotechniker heute oder in Zukunft in der Lage sein, Lebewesen $\mathrm{zu}$ erzeugen, die keinen Konstitutionsbedingungen bislang vorhandener Arten entsprechen, können wir diesen Vorgang als Eingriff in die Evolution, als Produktion einer neuen Art deuten. Das Vorkommen einer neuen Art mit neuen artspezifischen Konstitutionsbedingungen spricht aber nicht dagegen, daß die Zugehörigkeit von Individuen zur "alten" Art an die alten, stabilen Konstitutionsbedingungen gebunden ist.

\section{Annäherung an einen problematischen Begriff}

Ausgehend von den angegebenen Voraussetzungen können wir uns nun dem Begriff "Natürliche Arten" annähern. Die Voraussetzungen bestehen darin, daß sich Identitäts - und Existenzproblematik nicht losgelöst voneinander erörtern lassen, daß die Identität von Dingen sortal dependent ist sowie darin, daß diese sortale Dependenz auf artpezifischen Existenz-, Kontinuitäts- und Konstitutionsbedingungen beruht. Unsere Annäherung geht davon aus, daß es Vorkommnisse, wie Rosa, gibt, welche auf besondere Weise artspezifischen Bedingungen unterliegen. Guten Intuitionen nach ist es für ihr Vorkommen notwendig, dieser ihrer Art anzugehören. Ihr Vorkommen unterliegt Kontinuitätsbedingungen, die wohl kaum als Ergebnis konventioneller Festlegungen aufgefaßt werden können. Eine spezielle Kontinuitätsbedingung ist, daß ihre zeitliche Geschichte nicht vollständig unterbrochen werden kann. Ihr Vorkommen ist schließlich an artspezifische Konstitutionsbedingungen gebunden, die nicht nach praktisch-pragmatischen Kriterien technisch fortentwickelt werden können.

Ich schlage nun vor, derartige Vorkommnisse Vorkommnisse natürlicher Arten zu nennen.

Ebensowenig wie die genannten Bedingungen ausschließend oder exhaustiv sind, sind es die Elemente dieser Annäherung an den Begriff "natürliche Art". Der Aufweis dieser Elemente ist keine Lösung des Problems der Ontologie natürlicher Arten. Diese Überlegungen sollten lediglich einen Weg andeuten, der dazu führen kann, Vorkommnisse natürlicher Arten von solchen künstlicher oder artifizieller Arten (Artefakten) zu unterscheiden. Diese Unterscheidung wäre eine ontologische, da ja Fragen nach Existenz, raumzeitlicher Kontinuität und Konstitution zweifelsohne ontologische sind. Somit bekenne ich mich zu einem Realismus natürlicher Arten; jedoch zu einem mit einem "human face", der es versteht sich gegen beide eingangs erwähnte Verführungen zu wappnen. 
Armstrong 1989: Universals. Boulder/San Francisco/London.

Hirsch 1971: Essence and Identity. In: Munitz (ed.), Identity and Individuation. New York, 31-49.

Hirsch 1982: The Concept of Identity. Oxford.

Kanzian 1994: Der Begriff "Koinzidenz” in der Mereologie. In: Meggle/Wessels(Ed.), Analyomen1-Proceedings. Berlin/New York, 892-898.

Lowe 1989: Kinds of Being. Oxford.

Quine 1953: On What There Is. In: ders., From a Logical Point of View. Cambridge (Mass.), 1-19.

Wiggins 1980: Sameness and Substance. Oxford. 